\title{
A Prospective Observational Study to Evaluate the Severity Assessment Scores in Community-acquired Pneumonia for Adult Patients
}

\author{
Akhila Babu, Nybin Jose, Jona Jose \\ Department of Respiratory Therapy, School of Allied Health Sciences, Manipal University, Manipal, Udupi, Karnataka, India
}

\section{Abstract}

Introduction: Assessment of severity is the first step for determining whether a patient diagnosed with community-acquired pneumonia (CAP) needs to be admitted to the hospital or can be treated on outpatient basis. Aim: This study compares the ability of three severity scoring systems, systolic blood pressure, multilobar chest radiography involvement, albumin level, respiratory rate, tachycardia, confusion, oxygenation, and arterial pH (SMART-COP), confusion, urea nitrogen, respiratory rate, blood pressure less than 90/60 mm Hg and age over 65 years (CURB-65), and pneumonia severity index (PSI) to predict the need for mechanical ventilation and inotropic support among adult patients admitted to the hospital. Methodology: This was an observational study conducted on patients admitted from March 2016 to July 2016 to the Intensive Care Unit (ICU). Demographic data, severity scores from CURB-65, PSI, and SMART-COP, were documented. Patients were followed up for the need for mechanical ventilatory/inotropic support. The overall mortality of patients with CAP was recorded. Results: A total of eighty patients with CAP were included in this study. Forty-seven (59\%) were male. A CURB-65 severity score $\geq 2$ had a sensitivity, specificity, and negative predictive value (NPV) of $85.7 \%, 47.5 \%$, and $9.7 \%$, respectively, for ICU admission. For a PSI severity score $\geq 4$, the sensitivity, specificity, and NPV were $71.4 \%, 46.8 \%$, and $18.6 \%$. SMART-COP severity score $>3$ had a sensitivity, specificity, and NPV of $85.7 \%, 62.4 \%$, and 20.7\%, respectively. In predicting inotropic support, CURB-65 (PSI, SMART-COP) had sensitivity of $85.4 \%(80.5 \%, 90.2 \%)$, specificity of $64.1 \%(64.1 \%, 81.5 \%)$ and NPV of $19.4 \%(24.2 \%, 28.8 \%)$. Conclusions: SMART-COP scoring system is superior to CURB-65 and PSI in predicting the need for mechanical ventilation and inotropic support.

Keywords: Community-acquired infections/diagnosis, pneumonia, severity of illness index

\section{INTRODUCTION}

Pneumonia is an infection of the lung parenchyma. Community-acquired pneumonia (CAP), as the name suggests, is pneumonia acquired in the community unlike a hospital-acquired (nosocomial) pneumonia (HAP) which is acquired in the hospital. ${ }^{[1-3]}$ CAP is mainly due to the bacterial origin and has been traditionally classified as "typical" and "atypical." The typical pathogens include Streptococcus pneumoniae, Haemophilus influenzae, and Staphylococcus aureus. Atypical organisms such as Chlamydophila pneumoniae, Mycoplasma pneumoniae, and Legionella species are rarely identified in clinical practice because special laboratory tests are necessary for their identification. ${ }^{[4,5]}$ The clinical features of CAP are essentially that of pneumonia where the patients present

\begin{tabular}{|l|l|}
\hline \multicolumn{2}{|c|}{ Access this article online } \\
\hline Quick Response Code: & Website: \\
& www.ijrconline.org \\
\cline { 2 - 2 } & \\
\hline
\end{tabular}

with fever with chills and rigors, cough with sputum, chest discomfort or dyspnea. However, many patients who satisfy these criteria do not have pneumonia and could be having acute bronchitis. An important reason for overuse of antibiotics is inability to distinguish CAP from acute bronchitis. Furthermore, CAP may or may not present with fever with or without localizing features, which add morbidity and mortality in adults. A severity assessment is the first step for determining whether a patient with CAP can be treated safely as an outpatient or requires hospitalization

Address for correspondence: Ms. Akhila Babu, Department of Respiratory Therapy, School of Allied Health Sciences, Manipal University, Manipal, Udupi, Karnataka, India. E-mail: akhila.babu@manipal.edu

This is an open access article distributed under the terms of the Creative Commons Attribution-NonCommercial-ShareAlike 3.0 License, which allows others to remix, tweak, and build upon the work non-commercially, as long as the author is credited and the new creations are licensed under the identical terms.

For reprints contact: reprints@medknow.com

How to cite this article: Babu A, Jose N, Jose J. A prospective observational study to evaluate the severity assessment scores in community-acquired pneumonia for adult patients. Indian J Respir Care 2017;6:820-3. 
Babu, et al.: Severity assessment scores in community-acquired pneumonia

with or without Intensive Care Unit admission and selecting an antibiotic regimen. Hence, prediction rules have been developed to assist in the decision-making for care for CAP. The most commonly used prediction rules are the pneumonia severity index (PSI), confusion, urea nitrogen, respiratory rate, blood pressure less than $90 / 60 \mathrm{~mm} \mathrm{Hg}$ and age over 65 years (CURB-65), and systolic blood pressure, multilobar chest radiography involvement, albumin level, respiratory rate, tachycardia, confusion, oxygenation, and arterial $\mathrm{pH}$ (SMART-COP). The use of these tools is to assess the requirement of ventilator and vasopressor support. This study was done to evaluate severity scores such as SMART-COP, PSI, and CURB-65 for their ability to predict the need for mechanical ventilation and/inotropic support for adults patients admitted to the hospital with CAP.

\section{Methodology}

This observational study was conducted in patients admitted with CAP from March 2016 to July 2016. The study received ethical clearance. The patients who developed or had more $\geq 3$ symptoms such as productive cough, dyspnea, pleuritic chest pain, hemoptysis, fever $\left(>37.8^{\circ} \mathrm{C}\right)$, headache, and the presence of new infiltrates on the chest radiograph were included as study subjects. HAP (i.e., development of pneumonia $>48 \mathrm{~h}$ after hospitalization or discharge from another hospital $<2$ weeks before hospital admission), active thoracic malignancy, immunocompromised patients, pulmonary embolism, and patients who were in palliative care were excluded from the study. For all patients admitted to the hospital with CAP, a proforma was completed at the time of hospital admission. This included patient's vitals (blood pressure, heart rate, respiratory rate, temperature and pulse oximetry) and standard blood tests (i.e., full blood count, urea and electrolyte analysis, liver function tests, and assessment of C-reactive protein level). The reports were collected within 24 hours.

The severity assessment tools used were the CURB-65 score, PSI, and the SMART-COP scores, which were specifically designed to predict the need for intensive respiratory and/or vasopressor support. The scores were calculated and documented. The primary end point was a requirement for mechanical ventilation and/or inotropic support. These tools were used to predict the need of intensive respiratory, vasopressor support and to evaluate mortality for adult patients admitted with CAP. The decision to initiate mechanical ventilation and/or inotropic support was taken by the attending physicians. The overall outcome of patients with CAP was also assessed.

\section{Statistical analysis}

All data were compiled in Excel and transferred to SPSS for Windows, Version 16.0. Chicago, SPSS Inc, for analysis. Descriptive statistics are expressed as mean \pm standard deviation (SD) A $P<0.05$ or less was considered statistically significant. Sensitivity, specificity, positive predictive value, and negative predictive value (NPV) were calculated for different CURB-65, PSI, and SMART-COP grades with qualitative data (mechanical ventilation and inotropic support) as outcome.

\section{RESULTS}

Out of eighty patients included in the study, 47 (59\%) were male and $33(41 \%)$ female with mean $( \pm \mathrm{SD})$ age of $53( \pm 17)$ years. The major comorbidities of patients associated with CAP were chronic renal failure (40\%), congestive heart failure $(30 \%)$, and chronic liver failure $(25 \%)$ [Table 1 ]. A definite microbiological diagnosis was made for patients and streptococcus pneumonia was commonly seen to cause CAP [Table 2].

The sensitivity and specificity for CURB-65 were higher in Class 2 which is statistically significant and better prediction for mechanical ventilation and inotropic support [Table $3 a$ and $b$ ].

\begin{tabular}{lc}
\hline $\begin{array}{l}\text { Table 1: The comorbidities in patients admitted with } \\
\text { community-acquired pneumonia }\end{array}$ \\
\hline Diseases & Number of patients \\
\hline Neoplastic disease & 1 \\
Cerebrovascular disease & 7 \\
Chronic liver disease & 20 \\
Congestive cardiac failure & 24 \\
Chronic renal disease & 32 \\
\hline
\end{tabular}

Table 2: Microbiological diagnosis in patients admitted with community-acquired pneumonia

\begin{tabular}{lc}
\hline Organisms & Number of patients \\
\hline Adenovirus & 1 \\
S. aureus & 2 \\
E. coli & 3 \\
H. influenzae & 3 \\
Methicillin-resistant S. aureus & 5 \\
K. pneumoniae & 5 \\
Influenza virus (Type A and B) & 6 \\
S. pneumoniae & 8 \\
\hline S. aureus: Staphylococcus aureus, H. influenza: Haemophilus influenzae, \\
E. coli: Escherichia coli, S. pneumonia: Streptococcus pneumoniae, \\
K. pneumoniae: Klebsiella pneumoniae
\end{tabular}

Table 3a: Sensitivity, specificity, negative and positive predictive values for various classes of CURB-65 scoring system for predicting mechanical ventilation

\begin{tabular}{lcccc}
\hline $\begin{array}{l}\text { CURB-65 } \\
\text { class }\end{array}$ & Sensitivity (\%) & Specificity (\%) & NPV (\%) & PPV (\%) \\
\hline 2 & 85.7 & 47.5 & 9.7 & 63.3 \\
3 & 95.2 & 27.1 & 5.9 & 68.3 \\
\hline \multicolumn{4}{l}{ PPV: Positive predictive value, NPV: Negative predictive value, } \\
CURB-65: Confusion, urea nitrogen, respiratory rate, blood pressure less \\
than 90/60 mm Hg and age over 65 years
\end{tabular}


Babu, et al.: Severity assessment scores in community-acquired pneumonia

There was a statistically significant difference $(P<0.005)$ when sensitivity and specificity of scoring system were compared, PSI score was better in predicting need for mechanical ventilation and inotropic support [Table $4 \mathrm{a}$ and $\mathrm{b}$ ]. In SMART-COP, severity class 3 predicted the need for mechanical ventilation and inotropic support with high sensitivity, specificity [Table 5a and b].

It was also seen out of 80 patients, 59 patients required mechanical ventilation and 41 needed inotropic support out of their hospital stay. The majority of them fell into low severity in CURB-65 and PSI, whereas in SMART-COP, thirty patients

Table $3 \mathrm{~b}$ : Sensitivity, specificity, negative and positive
predictive values for various classes of CURB-65 scoring
system for predicting inotropic support

\begin{tabular}{lcccc}
\hline $\begin{array}{l}\text { CURB 65 } \\
\text { class }\end{array}$ & Sensitivity (\%) & Specificity (\%) & NPV (\%) & PPV (\%) \\
\hline 2 & 85.4 & 64.1 & 19.4 & 28.6 \\
3 & 100 & 20.3 & 5.9 & 36.5 \\
\hline
\end{tabular}

PPV: Positive predictive value, NPV: Negative predictive value, CURB-65: Confusion, urea nitrogen, respiratory rate, blood pressure over 65 years

\begin{tabular}{|c|c|c|c|c|}
\hline PSI class & Sensitivity (\%) & Specificity (\%) & NPV (\%) & PPV (\%) \\
\hline II & 61.9 & 67.8 & 16.7 & 59.4 \\
\hline III & 71.4 & 67.8 & 18.6 & 68.1 \\
\hline IV & 100 & 20.3 & 0 & 69.1 \\
\hline $\mathrm{V}$ & 100 & 20.3 & 0 & 69.1 \\
\hline
\end{tabular}

Table 4b: Sensitivity, specificity, negative, and positive predictive values for different PSI classes for predicting inotropic support

\begin{tabular}{lcccc}
\hline PSI class & Sensitivity (\%) & Specificity (\%) & NPV (\%) & PPV (\%) \\
\cline { 2 - 5 } II & 63.4 & 84.6 & 31.2 & 18.8 \\
III & 80.5 & 64.1 & 24.2 & 29.8 \\
IV & 100 & 30.8 & 0 & 39.7 \\
V & 100 & 30.8 & 0 & 39.7 \\
\hline \multicolumn{4}{l}{ PPV: Positive predictive value, NPV: Negative predictive value }
\end{tabular}

\begin{tabular}{|c|c|c|c|c|}
\hline \multicolumn{5}{|c|}{$\begin{array}{l}\text { Table 5a: Sensitivity, specificity, negative and positive } \\
\text { predictive values for various classes of SMART-COP scoring } \\
\text { system for predicting mechanical ventilation }\end{array}$} \\
\hline $\begin{array}{l}\text { SMART-COP } \\
\text { class }\end{array}$ & Sensitivity (\%) & Specificity (\%) & NPV (\%) & PPV (\%) \\
\hline 2 & 47.6 & 81.4 & 18.6 & 52.4 \\
\hline 3 & 85.7 & 62.4 & 20.7 & 65.4 \\
\hline 4 & 90.5 & 25.4 & 11.8 & 69.8 \\
\hline
\end{tabular}

PPV: Positive predictive value, NPV: Negative predictive value, SMART-COP: Systolic blood pressure, multilobar chest radiography involvement, albumin level, respiratory rate, tachycardia, confusion, oxygenation, and arterial $\mathrm{pH}$ were grouped as severe. From this study, it was shown that SMART-COP is a better predictive tool compared to CURB-65 and PSI in predicting mechanical ventilation and inotropic support. Nearly $75 \%$ of patients survived from community pneumonia and showed that early antibiotic therapy effectively cured the patients.

\section{Discussion}

It is vital that the clinician takes a correct diagnostic and prognostic decision. Prompt and accurate diagnosis and assessment of severity of illness guide further decision-making. The level of treatment received and overall cost of treatment signifies with it. CURB-65 is a validated method of predicting inpatient mortality associated with CAP that is recommended by the British Thoracic Society. ${ }^{[6]}$ The major breakthrough is the use of point system to score the risk.

Fine et al. developed a pneumonia severity index (PSI) score as part of the Pneumonia Patient Outcome Research Team study. ${ }^{[7]}$ The twenty items in the PSI included three demographic variables, five comorbid conditions, five physical examination findings, and seven laboratory/imaging results. Points were assigned and tallied for each variable present, and the final score was then broken into five risk classes. Patients in risk Classes I-III were considered low risk and manageable as outpatients, whereas classes IV and V might require hospitalization.

The "SMART-COP" is a simple tool that is the result of an extensive study on CAP called the Australian CAP Study. The tool was designed to overcome the limitations of PSI and CURB-65 to predict which patients will require intensive respiratory or vasopressor support. ${ }^{[8]}$ In our study, we found that SMART-COP gave a better prediction in mechanical ventilation and inotropic support because it had high sensitivity, specificity, and NPV such as $85.7 \%, 62.4 \%$, and $20.7 \%$ and $90.2 \%, 81.5 \%$, and $34.3 \%$, respectively. Inotropic support has higher values compared to mechanical ventilation because the majority of the patients required vasopressor support during therapy even though need of ventilation was minimal. The results were similar to the study done by Chalmers and Singanayagam ${ }^{[9]}$ in which SMART-COP was a better tool for predicting mechanical ventilation and inotropic support with sensitivity, specificity, and NPV of $84.8 \%, 82.1 \%$, and $98.1 \%$, respectively, compared to CURB 65 and PSI, thus proving that SMART-COP had performance characteristics superior to those of both CURB-65 and PSI. Both CURB-65 and PSI had low specificity and sensitivity in our study which was similar to the study done by Shah et al. ${ }^{[10]}$ They compared the death and admission as the primary outcomes. Overall mortality rate in patients treated for CAP, irrespective of comorbidities, was significantly lower with the use of proper antibiotic management at our hospital. 
Babu, et al.: Severity assessment scores in community-acquired pneumonia

\begin{tabular}{|c|c|c|c|c|}
\hline \multicolumn{5}{|c|}{$\begin{array}{l}\text { Table 5b: Sensitivity, specificity, negative and positive } \\
\text { predictive values for various classes of SMART-COP scoring } \\
\text { system for predicting inotropic support }\end{array}$} \\
\hline $\begin{array}{l}\text { SMART-COP } \\
\text { class }\end{array}$ & Sensitivity (\%) & Specificity (\%) & NPV (\%) & PPV (\%) \\
\hline 2 & 43.9 & 92.3 & 39 & 14.3 \\
\hline 3 & 90.7 & 81.5 & 34.3 & 28.8 \\
\hline 4 & 97.6 & 41 & 5.9 & 36.5 \\
\hline
\end{tabular}

PPV: Positive predictive value, NPV: Negative predictive value, SMART-COP: Systolic blood pressure, multilobar chest radiography involvement, albumin level, respiratory rate, tachycardia, confusion, oxygenation, and arterial $\mathrm{pH}$

\section{Conclusions}

CURB 65 and PSI scores, although widely used and validated for predicting mortality in large populations, are less accurate for prediction of outcomes such as the need for admission to the intensive care unit. The predictive value is good for SMART-COP is better than CURB-65 and PSI but may not identify some patients who require mechanical ventilation and inotropic support. Further studies are required with large population comparing different predictive tools available.

\section{Acknowledgment}

The authors would like to thank Mr. Ramesh Unnikrishnan, Assistant Professor (Senior Scale), Incharge of Department of Respiratory Therapy, for his assistance in manuscript preparation and review.

\section{Financial support and sponsorship}

Nil.

\section{Conflicts of interest}

There are no conflicts of interest.

\section{RefEREnCES}

1. Lim WS, Baudouin SV, George RC, Hill AT, Jamieson C, Le Jeune I, et al. BTS guidelines for the management of community acquired pneumonia in adults: Update 2009. Thorax 2009;64 Suppl 3:iii1-55.

2. Marrie TJ, Poulin-Costello M, Beecroft MD, Herman-Gnjidic Z. Etiology of community-acquired pneumonia treated in an ambulatory setting. Respir Med 2005;99:60-5.

3. Marik PE. The clinical features of severe community-acquired pneumonia presenting as septic shock. Norasept II Study Investigators. J Crit Care 2000;15:85-90.

4. Menon RU, George AP, Menon UK. Etiology and Anti-microbial sensitivity of organisms causing community acquired pneumonia: A single hospital study. J Family Med Prim Care 2013;2:244-9.

5. Shah BA, Singh G, Naik MA, Dhobi GN. Bacteriological and clinical profile of Community acquired pneumonia in hospitalized patients. Lung India 2010;27:54-7.

6. Community-acquired pneumonia in adults in British hospitals in 1982-1983: A survey of aetiology, mortality, prognostic factors and outcome. The British Thoracic Society and the Public Health Laboratory Service. Q J Med 1987;62:195-220.

7. Fine MJ, Auble TE, Yealy DM, Hanusa BH, Weissfeld LA, Singer DE, et al. A prediction rule to identify low-risk patients with community-acquired pneumonia. N Engl J Med 1997;336:243-50.

8. Charles PG, Wolfe R, Whitby M, Fine MJ, Fuller AJ, Stirling R, et al. SMART-COP: A tool for predicting the need for intensive respiratory or vasopressor support in community-acquired pneumonia. Clin Infect Dis 2008; $47: 375-84$.

9. Chalmers JD, Singanayagam A, Hill AT. Predicting the need for mechanical ventilation and/or inotropic support for young adults admitted to the hospital with community-acquired pneumonia. Clin Infect Dis 2008;47:1571-4.

10. Shah BA, Ahmed W, Dhobi GN, Shah NN, Khursheed SQ, Haq I. Validity of pneumonia severity index and CURB-65 severity scoring systems in community acquired pneumonia in an Indian setting. Indian J Chest Dis Allied Sci 2010;52:9-17. 